\title{
On the Effect of Outward Direct Investment on Home Country's Happiness
}

\author{
Wu Shujuan ${ }^{1, a, *}$, Xiao Jianhua ${ }^{1, b}$ \\ ${ }^{1}$ Economy Management College, Wuyi University, Yingbindadaozhong, Pengjiang, Jiangmen \\ a jane333444@126.com, b1312655857@qq.com \\ *corresponding author
}

Keywords: Outward direct investment, Happiness, Evaluation

\begin{abstract}
Based on the fact that input factors of production can not be completely replaced, a large number of outward direct investment leads to the fluctuation of jobs, income, growth of economy and inflation etc in home country. And these changes lead to the change of happiness of residents in home country to a large extent. Taking the case of China, using the Ordered Logit model, the paper analysis the effect of Chinese outward direct investment on the Chinese residents' happiness. Results show that Chinese outward direct investment and domestic residents' happiness are positively correlated, but the effect of outward direct investment on different people's happiness varies.
\end{abstract}

\section{Introduction}

The economic globalization is constantly evolving and promotes global investment. Fast development of international investmeent raises hot debate on the effect of outward direct investment(ODI) on the home country's economy, science and technology, employment and other aspects. Lots of studies on the ODI's effect in developed countires, like US, Japan, etc, have been carried out by scholars all over the world. However, current studies have not attach enough importance to the effect of ODI on the happiness of home country's residents, as the ultimate goal of human activities is to obtain happiness. According to classical economics principle, the input factors of production can not be completely replaced. a large number of capital transfer affects jobs, incomes, economic growth, inflation, etc. And all these changes of factors lead to fluctuation of happiness in home country to a large extent. So, more attention should be paid to the effect of ODI on happinessof home coutry's residents. Based on this, this paper takes China as the object of study, explores the impact of China's ODI on the Chinese residents' happiness.

\section{Theoretical Basis}

\subsection{Economy is an Important Index Affecting Happiness}

National happiness is proposed firstly by Jigme Singh Wangchuck, Bhutan's fourth king, in 1972. Since then, the west has carried out a large number of practise and theoretical studies on happiness, like OECD Better Life Index(BLI), the United Nations global happiness index, Bhutan national happiness index, Japan happiness index, hexun.com happy index, the Kunming happiness index system, the Guangdong happy index system, Zhejiang happy index system, Chenzhou city happiness index, ect.

The above index systems show that, the existing happiness index systems basically contain 
economic indicators, health indicators, environmental indicators, civilization and welfare indicators. Economic well-being embodied in the economic indicators is the material basis and the basic conditions for happiness. Economic indicators, including GDP per capita, disposable income per capita, Engel coefficient, the level of unemployment, household consumption rate, CPI, Gini coefficient and social security coverage and other two indicators are objective conditions for economic well-being.

\subsection{ODI Affects Domestic Economic Factors}

According to economic theory, ODI influences employment, income, economic growth, inflation and other economic factors in home country. The effect of ODI to the economic factors is complex and combined with negative effect and positive effects.

The effect of ODI on the employment lies in many aspects, including the total amount of employment, the industry allocation of employment, and district allocation of employment, ect. What worthes mentioning is that some effect is controvertial. But it is generally agreed that, with the outward trandfer of capital, ie ODI, the employment opportunity is getting less in home country, which means the increase of the unemployment rate. As for the structure of employment, it varies with industries and also the technical contents of industries. If ODI is substitive between host country and home country, it would harm home country workers. Or, if it is complementary, it is beneficial to home country workers. And the higher technology-intensive an industry is, the more employment opportunities will be created, while the lower technology-intensive an industry is, the less employment opportunities will be.

ODI does not only affect the income of workers in home country, and also the income gap. ODI has a significant impact on the wage ${ }^{[1]}$, and presents a "U" type trend ${ }^{[2]}$. Enterprises of ODI significantly increase the average wage level of employees, and the positive wage spillover effect is persistent and increasing ${ }^{[3-4]}$. Mao's study also confirms salary of the overseas staff is upgraded. ODI enterprises have higher the wage payroll than non-ODI enterprise. Compared to the central and western China, the impact in ODI enterprises in the eastern China is smaller. The influence of ODI enterprises on the wage level is from the productivity effect[5]. ODI also significantly increases the wage gap within the enterprise ${ }^{[3]}$. Slaughter (1993) and others believe that the popularization of computer and related technologies in the United States tend to employ technology-intensive workers, which lead to the expansion of wage gaps between the skilled technical workers and unskilled workers in United States ${ }^{[6]}$. The results of the study in Taiwan and Singapore also confirm the above opinion ${ }^{[7]}$. Qi Jianmei (2017) confirms the hypothesis that there is a nonlinear dynamic effect of ODI on the wage gap between Chinese enterprises[8].

ODI also affects home country's economic growth. IDP (Investment Development Path) theory proposed by Dunning (1981) is considered as the cornerstone of the theory of ODI and economic growth $^{[9]}$. According to the IDP theory, ODI of a country (or region) relates to the net position of ODI and home country economic growth level. The higher economic growth is, the larger ODI is. Pichl(1989), based on the empirical study of the developed countries in the world, agrees with Dunning's view that the scale of a country's ODI depends on the level of economic development ${ }^{[10]}$. Since then, many scholars have carried out theoretical research and empirical research on the way(like reference [11]) and the IDP phase of the countries[12-15].

The impact of ODI on home country inflation. Studies on the relationship between home country's inflation rate and ODI are still in the primary stage. The current study shows that the two are correlated. There is a negative correlation between ODI and the inflation rate ${ }^{[16-17]}$.

In summary, effects of ODI on macroeconomic factors in home country may be of positive effect, or negative effect. The overall effect depends on the degree of impact of ODI on these factors, the weight and the degree of influence of these factors on people's happiness in home country. So the 
effect of ODI on the happiness of home country people can be negative or positive. The total effect is the offset of the two effects. Different groups have different effects.

\section{Empirical Test}

\subsection{Building Model}

The calculation methods of national happiness are various, and there is no uniform formula and method. Referring to the study of Kanweri(2015) ${ }^{[18]}$ and Tella RD, et al(2007) ${ }^{[19]}$ and other related researches, this paper uses Ordered Logit Model to analyze the effect of ODI on the happiness of residents in home country as follows:

$$
\text { Happy }_{\text {st }}=\mathrm{a}_{1} O D I_{s t}+\mathrm{a}_{2} M A C R O_{\text {st }}+a_{3} M I C R O_{\text {ist }}+\eta_{s}+\mu_{t}+\varepsilon_{\text {ist }}
$$

In the model (1), the explanatory variable, Happyist, indicates the subjective well-being in $s$ province in year t. The independent variable $O D I_{s t}$ indicates the s province's ODI in year t.

The independent variables $M A C R O_{s t}$ and $M I C R O_{i s t}$ indicate the macroeconomic variables that can affect the individual's happiness and the micro level variables of individual happiness respectively. According to the "happiness gap" concept in the "happiness gap"[20], Gini coefficient, increase of annual income, rate of inflation and unemployment are taken as factors.

As for the $M I C R O_{i s t}$, gender(SEX), age $(A G E)$, education level(EDU), marital status(MARI), employment status(EMPL), self-rated health (HEALS), and the type of work(PRO), family income level (INCOMS) are taken as factors. $\eta_{S}$ indicates dummy variable. $\varepsilon_{i s t}$ indicates the error term of the regression model.

\subsection{Data and Data Description}

ODI data is from the Ministry of Commerce "going out" enterprises directory data base in 2002, 2008, 2014. The datas of INCOM, GINI, INFLA, UNEMP are from "Chinese Statistical Yearbook". Data of Happyist are from the World Values Survey (WVS). The descriptive statistics of specific indicators and data sources are shown in table 1.

\subsection{Model Estimation}

Using Stata12.0, applying the Ordered Logit model maximum likelihood method, we carry out an regression analysis. The results are shown in table 2. Part (1) of the table is the result of the regression of $\mathrm{FOH}$ and the independent variables repectively, part (2) is the result of regression(1) plus independent variable $O D I$, part (3) is the result of regression (2) plus independent variable $\triangle I N C O M$, part (4) is the result of regression (2) plus independent variable GINI, part (5) is the result of regression (2) plus independent variables $\triangle I N C O M$ and GINI, part (6) is the result of regression (5) plus independent variable INFLA, part (7) is the result of regression (6) plu independent variable UNEMP. 
Table 1 Data Description

\begin{tabular}{|c|c|c|c|c|c|c|}
\hline Index & $\begin{array}{c}\text { 2nd degree } \\
\text { In }\end{array}$ & description & Average & $\begin{array}{c}\text { Standard } \\
\text { error }\end{array}$ & Minimum & Maximum \\
\hline Happy & FOHst & $\begin{array}{c}\text { 1: very unhappy; 4: very } \\
\text { happy }\end{array}$ & 3.009893 & 0.5997 & 1 & 4 \\
\hline ODI & ODIst & $\begin{array}{c}\text { Numbers of enterprises } \\
\text { invested aboad in a province }\end{array}$ & 27.8749 & 36.2173 & 0 & 156 \\
\hline Macro & AINCOMst & $\begin{array}{c}\text { Average income of workers in } \\
\text { town in a province }\end{array}$ & 4503.038 & 1519.401 & 2547 & 10021 \\
\cline { 2 - 7 } & GINIst & Gini coifficient in a province & 0.397539 & 0.0569 & 0.1965 & 0.496 \\
\cline { 2 - 7 } & INFLAst & Inflation rate in a province & 102.5828 & 1.5123 & 99.7 & 108.5 \\
\cline { 2 - 7 } & UNEMPst & $\begin{array}{c}\text { Unemployment rate in a } \\
\text { province }\end{array}$ & 7.501514 & 11.0489 & 1.3 & 54.36 \\
\hline Micro & SEXist & 1: maile; 2: femail & 1.433636 & 0.4958 & 1 & 2 \\
\cline { 2 - 7 } & AGEist & Note & 3.379736 & 1.4747 & 1 & 6 \\
\cline { 2 - 7 } & EDUist & 1: low; 2: midium; 3: high & 1.885408 & 0.6391 & 1 & 3 \\
\cline { 2 - 7 } & MARIist & Note & 1.965375 & 0.5618 & 1 & 5 \\
\cline { 2 - 7 } & EMPList & Note & 4.830173 & 1.4325 & 1 & 8 \\
\cline { 2 - 7 } & HEALSist & $1:$ very bad; : very good & 3.875618 & 0.8691 & 2 & 5 \\
\cline { 2 - 7 } & INCOMSist & Note & 1.339374 & 1.2496 & 1 & 7 \\
\cline { 2 - 7 } & PROist & Note & 2.992593 & 0.7802 & 1 & 7 \\
\hline
\end{tabular}

Note: [1]Age: 1:15-24; 2:25-34; 3:35-44; 4:45-54; 5:55-64; 6:65--; [2]MARI: 1: married, 2: divorced; , 3: widow, 4: single, 5: living together; [3]EMP: 1: others, 2: house wife, 3: unemployment, 4: part time, 5: full time, 6: self-employed, 7: retiared, 8: students; [4]INCO: 1: Below5000, 2: 5000-10000, 3:10,000-20,000, 4: 20,000-50,000, 5: 50,000-100,000, 6: 100,000; [5]: PRO:1: Agriculture or farmer; 2: employer, 3: unskilled, 4: semi-skilled; 5: skilled, 6: professional, 7: others.

Table 2 Result of the Regressions

\begin{tabular}{|c|c|c|c|c|c|c|c|}
\hline & (1) & (2) & (3) & (4) & (5) & (6) & (7) \\
\hline$O D I$ & $\begin{array}{c}0.0046809 * * * \\
(.0016129) \\
\end{array}$ & & $\begin{array}{l}.0040921^{* *} \\
(.0016457)\end{array}$ & $\begin{array}{c}.0022803 \\
(.0017395)\end{array}$ & $\begin{array}{c}.0013833 \\
(.0017791) \\
\end{array}$ & $\begin{array}{c}.0011259 \\
(.0017879) \\
\end{array}$ & \\
\hline \multicolumn{8}{|c|}{ A: MACRO } \\
\hline$\triangle I N C O M$ & $\begin{array}{c}-.0000697^{* * *} \\
(.0000402) \\
\end{array}$ & $\begin{array}{l}-.0000572 * \\
(.0000406)\end{array}$ & $\begin{array}{c}-.0000909^{* * *} \\
(.0000416) \\
\end{array}$ & & $\begin{array}{c}-.0001061^{* * *} \\
(.0000421) \\
\end{array}$ & $\begin{array}{c}-.0001004^{* *} \\
(.0000422) \\
\end{array}$ & \\
\hline GINI & $\begin{array}{c}-4.626242^{* * *} \\
(1.0748)\end{array}$ & $\begin{array}{c}-4.074504 * * * \\
(1.123487)\end{array}$ & $\begin{array}{c}-4.582523^{* * *} \\
(1.147426)\end{array}$ & $\begin{array}{c}-4.495765^{* * *} \\
(1.145512)\end{array}$ & $\begin{array}{c}-5.186384^{* * *} \\
(1.179013)\end{array}$ & $\begin{array}{c}-5.284619^{* * *} \\
(1.180609)\end{array}$ & \\
\hline INFLA & $\begin{array}{l}-.0599835 * \\
(.0405099) \\
\end{array}$ & $\begin{array}{l}-.0440156 \\
(.0410331) \\
\end{array}$ & $\begin{array}{l}-.0514533 \\
(.0413826) \\
\end{array}$ & $\begin{array}{c}-.0773175 * * \\
(.0418085) \\
\end{array}$ & $\begin{array}{c}-.0948816 * * \\
(.0424571) \\
\end{array}$ & $\begin{array}{c}-.0978352^{* *} \\
(.0424965) \\
\end{array}$ & \\
\hline UNEMP & $\begin{array}{l}-.0085969 * \\
(.0053726)\end{array}$ & $\begin{array}{l}-.0072894 \\
(.0054103) \\
\end{array}$ & $\begin{array}{l}-.0065103 \\
(.005447) \\
\end{array}$ & $\begin{array}{l}-.0086692 \\
(.0054449)\end{array}$ & $\begin{array}{c}-.0076174^{* *} \\
(.0054793) \\
\end{array}$ & $\begin{array}{c}-.0082296^{* *} \\
(.0055054) \\
\end{array}$ & \\
\hline \multicolumn{8}{|l|}{ B: MICRO } \\
\hline \multicolumn{8}{|c|}{ SEX [MALE for reference] } \\
\hline FEMALE & $\begin{array}{c}.1697713 \\
(.1214106)\end{array}$ & $\begin{array}{l}.1599109 \\
.(1215046)\end{array}$ & $\begin{array}{c}.1580391 \\
(.1215231)\end{array}$ & $\begin{array}{c}.1708957 \\
(.1216113)\end{array}$ & $\begin{array}{c}.1690407 \\
.1216632)\end{array}$ & $\begin{array}{l}.1198886 \\
(.124186)\end{array}$ & $\begin{array}{r}.1206105 \\
(.1241946) \\
\end{array}$ \\
\hline ODI & & $\begin{array}{l}.004622^{* * *} \\
(.0016145) \\
\end{array}$ & $\begin{array}{c}.0043581^{* * *} \\
(.0016254 \\
\end{array}$ & $\begin{array}{l}.0028327 * \\
(.0017028) \\
\end{array}$ & $\begin{array}{c}.0021925 \\
(.0017299) \\
\end{array}$ & $\begin{array}{c}.0014052 \\
(.0017798) \\
\end{array}$ & $\begin{array}{r}.0011472 \\
(.0017885) \\
\end{array}$ \\
\hline \multicolumn{8}{|c|}{ AGE [15-24 for reference] } \\
\hline $25-34$ & $\begin{array}{c}-.02236 \\
(.2273486) \\
\end{array}$ & $\begin{array}{c}.009321 \\
(.2274641) \\
\end{array}$ & $\begin{array}{c}.0059893 \\
(.2277018)\end{array}$ & $\begin{array}{r}-.0040008 \\
(.227791) \\
\end{array}$ & $\begin{array}{l}-.0118771 \\
(.2281707) \\
\end{array}$ & $\begin{array}{c}.0139421 \\
(.2285619)\end{array}$ & $\begin{array}{c}.0177103 \\
(.2285299) \\
\end{array}$ \\
\hline $35-44$ & $\begin{array}{l}-.0643629 \\
(.2188754)\end{array}$ & $\begin{array}{l}-.0406338 \\
(.219044)\end{array}$ & $\begin{array}{l}-.0651102 \\
(.2198962)\end{array}$ & $\begin{array}{l}-.0453716 \\
(.2194478)\end{array}$ & $\begin{array}{l}-.0863286 \\
(.2205565)\end{array}$ & $\begin{array}{l}-.0546844 \\
(.2211754)\end{array}$ & $\begin{array}{l}-.0452366 \\
(.2213251) \\
\end{array}$ \\
\hline
\end{tabular}




\begin{tabular}{|c|c|c|c|c|c|c|c|}
\hline $45-54$ & $\begin{array}{c}.0160679 \\
(.2265665)\end{array}$ & $\begin{array}{l}.0609949 \\
(.227136) \\
\end{array}$ & $\begin{array}{c}.0371949 \\
(.2278653) \\
\end{array}$ & $\begin{array}{c}.0331266 \\
(.2274615) \\
\end{array}$ & $\begin{array}{l}-.0106426 \\
(.2285842)\end{array}$ & $\begin{array}{l}.0171508 \\
(.229093) \\
\end{array}$ & $\begin{array}{c}.0215726 \\
(.2291192) \\
\end{array}$ \\
\hline $55-64$ & $\begin{array}{l}-.1912168 \\
(.2348779)\end{array}$ & $\begin{array}{l}-.1374135 \\
(.235558)\end{array}$ & $\begin{array}{l}-.1558707 \\
(.2361534)\end{array}$ & $\begin{array}{l}-.1534738 \\
(.2357011)\end{array}$ & $\begin{array}{l}-.1868978 \\
(.236602) \\
\end{array}$ & $\begin{array}{l}-.1656995 \\
(.2368371)\end{array}$ & $\begin{array}{l}-.1412399 \\
(.2374261)\end{array}$ \\
\hline $65-$ & $\begin{array}{c}.26466 \\
(.2702627)\end{array}$ & $\begin{array}{c}.3221916 \\
(.2710882)\end{array}$ & $\begin{array}{c}.3107671 \\
(.2710827)\end{array}$ & $\begin{array}{c}.3097069 \\
(.2717292)\end{array}$ & $\begin{array}{c}.2868736 \\
(.2719443)\end{array}$ & $\begin{array}{c}.2943761 \\
(.2721126)\end{array}$ & $\begin{array}{c}.3142123 \\
(.2724991)\end{array}$ \\
\hline$O D I$ & & $\begin{array}{l}.0047498^{* *} \\
(.0016209) \\
\end{array}$ & $\begin{array}{l}.0044629 * * \\
(.0016328) \\
\end{array}$ & $\begin{array}{c}.002973 * \\
(.0017096) \\
\end{array}$ & $\begin{array}{c}.0022874 \\
(.0017392)\end{array}$ & $\begin{array}{c}.0014346 \\
(.0017891)\end{array}$ & $\begin{array}{c}.0011972 \\
(.0017967)\end{array}$ \\
\hline \multicolumn{8}{|c|}{$E D U$ [ LOW for reference] } \\
\hline MIDDLE & $\begin{array}{l}.255947^{* * *} \\
(.1418131) \\
\end{array}$ & $\begin{array}{l}.240814 * * \\
(.1419264) \\
\end{array}$ & $\begin{array}{l}.2547749 * * \\
(.1423046) \\
\end{array}$ & $\begin{array}{l}.2077738^{* *} \\
(.1422935) \\
\end{array}$ & $\begin{array}{c}.2213193^{* *} \\
(.142654) \\
\end{array}$ & $\begin{array}{l}.2218399 * * \\
(.1426816) \\
\end{array}$ & $\begin{array}{l}.2082472 * * \\
(.1431346) \\
\end{array}$ \\
\hline HIGH & $\begin{array}{c}.5651962 * * * \\
(.1926751) \\
\end{array}$ & $\begin{array}{l}.511922 * * * \\
(.1936173) \\
\end{array}$ & $\begin{array}{c}.5897761^{* * *} \\
(.197942) \\
\end{array}$ & $\begin{array}{c}.5153109 * * * \\
(.1932646) \\
\end{array}$ & $\begin{array}{c}.6309094 * * * \\
(.1980389) \\
\end{array}$ & $\begin{array}{c}.6198492^{* * *} \\
(.1981311) \\
\end{array}$ & $\begin{array}{c}.6051896 * * * \\
(.1985363) \\
\end{array}$ \\
\hline ODI & & $\begin{array}{c}.0042446 * * * \\
(.0016257) \\
\end{array}$ & $\begin{array}{c}.0037919 * * * \\
(.0016426) \\
\end{array}$ & $\begin{array}{c}.0024684 \\
(.001713) \\
\end{array}$ & $\begin{array}{c}.0014984 \\
(.0017512) \\
\end{array}$ & $\begin{array}{c}.0006624 \\
(.0017996) \\
\end{array}$ & $\begin{array}{c}.000448 \\
(.0018072)\end{array}$ \\
\hline \multicolumn{8}{|c|}{ Mari [MARRIED for reference] } \\
\hline DIVORCED & $\begin{array}{c}.5898786 * * \\
(.1828989) \\
\end{array}$ & $\begin{array}{c}.5979942 * * * \\
(.1827358) \\
\end{array}$ & $\begin{array}{l}.581286 * * * \\
(.1833771) \\
\end{array}$ & $\begin{array}{c}.5690992^{* * *} \\
(.1828093) \\
\end{array}$ & $\begin{array}{c}.5372166^{* * *} \\
(.1835099) \\
\end{array}$ & $\begin{array}{c}.5591941 * * * \\
(.1840912) \\
\end{array}$ & $\begin{array}{c}.5615608^{* * *} \\
(.184119) \\
\end{array}$ \\
\hline WIDOWED & $\begin{array}{c}.4742438 \\
(.3788921)\end{array}$ & $\begin{array}{c}.5343922 \\
(.3805862) \\
\end{array}$ & $\begin{array}{c}.512057 \\
(.3814583) \\
\end{array}$ & $\begin{array}{c}.5048706 \\
(.3809534) \\
\end{array}$ & $\begin{array}{c}.462345 \\
(.3819614) \\
\end{array}$ & $\begin{array}{c}.4713812 \\
(.3829642) \\
\end{array}$ & $\begin{array}{c}.4350476 \\
(.3841893)\end{array}$ \\
\hline SINGLE & $\begin{array}{l}2.145431 * \\
(1.384025)\end{array}$ & $\begin{array}{l}2.123657 * \\
(1.383072) \\
\end{array}$ & $\begin{array}{c}2.151522 \\
(1.383683)\end{array}$ & $\begin{array}{c}1.959584 \\
(1.387405) \\
\end{array}$ & $\begin{array}{c}1.992622 \\
(1.389656)\end{array}$ & $\begin{array}{c}1.951569 \\
(1.390616)\end{array}$ & $\begin{array}{c}1.907085 \\
(1.390667)\end{array}$ \\
\hline LIVING T & $\begin{array}{l}-.4829166 \\
(.4796797) \\
\end{array}$ & $\begin{array}{l}-.4715819 \\
(.4826813) \\
\end{array}$ & $\begin{array}{l}-.4568488 \\
(.4823281) \\
\end{array}$ & $\begin{array}{l}-.6003412 \\
(.4846624) \\
\end{array}$ & $\begin{array}{l}-.5884374 \\
(.4833185) \\
\end{array}$ & $\begin{array}{l}.5759663 \\
(.4833336) \\
\end{array}$ & $\begin{array}{l}.5978684 \\
(.4840966) \\
\end{array}$ \\
\hline$O D I$ & & $\begin{array}{c}.0047301^{* * *} \\
(.0016136) \\
\end{array}$ & $\begin{array}{c}.0045219 * * * \\
(.0016256) \\
\end{array}$ & $\begin{array}{c}.002972 * \\
(.0017007) \\
\end{array}$ & $\begin{array}{c}.0024099 \\
(.0017298) \\
\end{array}$ & $\begin{array}{c}.0014721 \\
(.0017808) \\
\end{array}$ & $\begin{array}{c}.0011892 \\
(.0017902) \\
\end{array}$ \\
\hline \multicolumn{8}{|c|}{ EMPL [ OTHER for reference] } \\
\hline HOUSWIF & $\begin{array}{c}.7761424^{* * *} \\
(.4215836) \\
\end{array}$ & $\begin{array}{l}.7666188^{* *} \\
(.4216069) \\
\end{array}$ & $\begin{array}{l}.7914103^{* *} \\
(.4220785) \\
\end{array}$ & $\begin{array}{l}.7434783^{* *} \\
(.4229461) \\
\end{array}$ & $\begin{array}{l}.7755241^{* *} \\
(.4235376) \\
\end{array}$ & $\begin{array}{c}.6920807 \\
(.4237839) \\
\end{array}$ & $\begin{array}{c}.6827485 \\
(.4239335) \\
\end{array}$ \\
\hline UNEMPED & $\begin{array}{l}-.693708^{*} \\
(.4905497) \\
\end{array}$ & $\begin{array}{l}-.6685337 \\
(.4908747) \\
\end{array}$ & $\begin{array}{l}.6090284 \\
(.4920472) \\
\end{array}$ & $\begin{array}{c}.8349845^{*} \\
(.4932291) \\
\end{array}$ & $\begin{array}{l}-.7653702 \\
(.4934319) \\
\end{array}$ & $\begin{array}{l}-.8026268 \\
(.4914029) \\
\end{array}$ & $\begin{array}{l}.8205514 * \\
(.4919595) \\
\end{array}$ \\
\hline PTTIM & $\begin{array}{c}.1681642 \\
(.3733248) \\
\end{array}$ & $\begin{array}{c}.1685711 \\
(.3733427) \\
\end{array}$ & $\begin{array}{c}.1744266 \\
(.3736934) \\
\end{array}$ & $\begin{array}{c}.137918 \\
(.3740308)\end{array}$ & $\begin{array}{c}.1424008 \\
(.3745152) \\
\end{array}$ & $\begin{array}{c}.0382631 \\
(.3765811) \\
\end{array}$ & $\begin{array}{c}.0381749 \\
(.3767193) \\
\end{array}$ \\
\hline FULTIM & $\begin{array}{c}.0796429 \\
(.3523877)\end{array}$ & $\begin{array}{c}.0442342 \\
(.3526462)\end{array}$ & $\begin{array}{c}.0786947 \\
(.3537061)\end{array}$ & $\begin{array}{c}.004264 \\
(.3534807) \\
\end{array}$ & $\begin{array}{l}.0507022 \\
(.354601)\end{array}$ & $\begin{array}{l}-.0006802 \\
(.3537619)\end{array}$ & $\begin{array}{c}.0139161 \\
(.3540302)\end{array}$ \\
\hline SELFEMP & $\begin{array}{c}.5343813 \\
(.4507346) \\
\end{array}$ & $\begin{array}{c}.4908559 \\
(.4513705) \\
\end{array}$ & $\begin{array}{c}.5380602 \\
(.4525754) \\
\end{array}$ & $\begin{array}{c}.4207817 \\
(.4526502) \\
\end{array}$ & $\begin{array}{c}.4824029 \\
(.4538712) \\
\end{array}$ & $\begin{array}{c}.4741899 \\
(.4523713) \\
\end{array}$ & $\begin{array}{c}.4718174 \\
(.4524714) \\
\end{array}$ \\
\hline RETIRED & $\begin{array}{c}.3552462 \\
(.3899897) \\
\end{array}$ & $\begin{array}{c}.3600714 \\
(.3902234) \\
\end{array}$ & $\begin{array}{c}.4360692 \\
(.3934274) \\
\end{array}$ & $\begin{array}{c}.2447361 \\
(.3920894) \\
\end{array}$ & $\begin{array}{c}.3429337 \\
(.3948361) \\
\end{array}$ & $\begin{array}{c}.262168 \\
(.3952693) \\
\end{array}$ & $\begin{array}{c}.299546 \\
(.3960423) \\
\end{array}$ \\
\hline STUD & $\begin{array}{c}.4207915 \\
(.4656747)\end{array}$ & $\begin{array}{c}.3203664 \\
(.4661874)\end{array}$ & $\begin{array}{c}.3855821 \\
(.4681267) \\
\end{array}$ & $\begin{array}{l}.2945894 \\
(.466654) \\
\end{array}$ & $\begin{array}{l}.3868822 \\
(.468419) \\
\end{array}$ & $\begin{array}{c}.3006742 \\
(.4690143)\end{array}$ & $\begin{array}{l}.2953655 \\
(.469149) \\
\end{array}$ \\
\hline ODI & & $\begin{array}{c}.0046751^{* * *} \\
(.0016344) \\
\end{array}$ & $\begin{array}{c}.0040751^{* * *} \\
(.0016334) \\
\end{array}$ & $\begin{array}{c}.0027595 \\
(.0017276) \\
\end{array}$ & $\begin{array}{c}.0020762 \\
(.0017561) \\
\end{array}$ & $\begin{array}{c}.0012137 \\
(.0018125) \\
\end{array}$ & $\begin{array}{c}.0009491 \\
(.0018214) \\
\end{array}$ \\
\hline \multicolumn{8}{|c|}{ HEALS [BAD for reference] } \\
\hline VERYBAD & -- & -- & -- & -- & -- & -- & -- \\
\hline FAIR & $\begin{array}{l}.738162 * * * \\
(.2612121) \\
\end{array}$ & $\begin{array}{c}.7156241^{* * *} \\
(.2615532) \\
\end{array}$ & $\begin{array}{l}.719883 * * * \\
(.2620952) \\
\end{array}$ & $\begin{array}{c}.7103928^{* * *} \\
(.2616649) \\
\end{array}$ & $\begin{array}{c}.7241906 * * * \\
(.2621296) \\
\end{array}$ & $\begin{array}{c}.6945843^{* * *} \\
(.2636068) \\
\end{array}$ & $\begin{array}{c}.6926016^{* * *} \\
(.2636465) \\
\end{array}$ \\
\hline GOOD & $\begin{array}{c}1.617899 * * * \\
(.256521) \\
\end{array}$ & $\begin{array}{c}1.589777^{* * *} \\
(.2570321) \\
\end{array}$ & $\begin{array}{c}1.590045^{* * *} \\
(.2570109) \\
\end{array}$ & $\begin{array}{c}1.589128^{* * *} \\
(.2571623) \\
\end{array}$ & $\begin{array}{c}1.590408^{* * *} \\
(.2570968) \\
\end{array}$ & $\begin{array}{c}1.56916 * * * \\
(.2585167) \\
\end{array}$ & $\begin{array}{c}1.566953^{* * *} \\
(.2585648) \\
\end{array}$ \\
\hline VERYGOOD & $\begin{array}{c}3.322789 * * * \\
(.2823372) \\
\end{array}$ & $\begin{array}{c}3.284948^{* * *} \\
(.2831917) \\
\end{array}$ & $\begin{array}{c}3.284405^{* * *} \\
(.2831756) \\
\end{array}$ & $\begin{array}{c}3.249532^{* * *} \\
(.283562) \\
\end{array}$ & $\begin{array}{c}3.245082 * * * \\
(.28352) \\
\end{array}$ & $\begin{array}{c}3.246337^{* * *} \\
(.2849257) \\
\end{array}$ & $\begin{array}{c}3.239575^{* * *} \\
(.2853165) \\
\end{array}$ \\
\hline ODI & & $\begin{array}{l}.0028129 * \\
(.0017194)\end{array}$ & $\begin{array}{c}.0027657 \\
(.0017299) \\
\end{array}$ & $\begin{array}{l}.0015616 \\
(.001799) \\
\end{array}$ & $\begin{array}{l}.0012908 \\
(.0018281 \\
\end{array}$ & $\begin{array}{c}.0001048 \\
(.0018794)\end{array}$ & $\begin{array}{c}.0000326 \\
(.0018868)\end{array}$ \\
\hline
\end{tabular}




\begin{tabular}{|c|c|c|c|c|c|c|c|}
\hline $5000-10000$ & $\begin{array}{c}.132339 \\
(.5050013)\end{array}$ & $\begin{array}{c}.2326496 \\
(.5072887)\end{array}$ & $\begin{array}{c}.162587 \\
(.5117885)\end{array}$ & $\begin{array}{c}-.039411 \\
(.5203679)\end{array}$ & $\begin{array}{l}-.1988955 \\
(.5277863)\end{array}$ & $\begin{array}{l}.1649389 \\
(.570829) \\
\end{array}$ & $\begin{array}{c}.1879569 \\
(.5736372)\end{array}$ \\
\hline $10,000-20,000$ & $\begin{array}{l}-.8894697 \\
(.6788087)\end{array}$ & $\begin{array}{l}-.8482527 \\
(.6805999)\end{array}$ & $\begin{array}{l}-.8771816 \\
(.6780287)\end{array}$ & $\begin{array}{c}-1.195481 * \\
(.6921167) \\
\end{array}$ & $\begin{array}{c}-1.303816 * \\
(.6889404)\end{array}$ & $\begin{array}{l}-1.042252 \\
(.7094413)\end{array}$ & $\begin{array}{l}-1.068453 \\
(.7104821)\end{array}$ \\
\hline 20,000-50,000 & $\begin{array}{c}.9497048^{* * *} \\
(.4977769) \\
\end{array}$ & $\begin{array}{c}-.8896909 * * \\
(.4981014) \\
\end{array}$ & $\begin{array}{c}-.9518261^{* *} \\
(.4996484) \\
\end{array}$ & $\begin{array}{c}-.9812761^{* *} \\
(.4968146)\end{array}$ & $\begin{array}{c}-1.099299 * * \\
(.4987897) \\
\end{array}$ & $\begin{array}{l}-.7885322 \\
(.5345176) \\
\end{array}$ & $\begin{array}{l}-.7623208 \\
(.5362205) \\
\end{array}$ \\
\hline $\begin{array}{l}50,000-100,00 \\
0\end{array}$ & $\begin{array}{c}.3806085 \\
(.7418263)\end{array}$ & $\begin{array}{l}.4695457 \\
(.744706)\end{array}$ & $\begin{array}{c}.5578329 \\
(.7442512)\end{array}$ & $\begin{array}{c}.1550235 \\
(.7611066)\end{array}$ & $\begin{array}{c}.2597397 \\
(.7603708)\end{array}$ & $\begin{array}{c}.5508081 \\
(.7856255)\end{array}$ & $\begin{array}{c}.544452 \\
(.7864541)\end{array}$ \\
\hline $100,000-$ & $\begin{array}{c}.0145008 \\
(.3417585) \\
\end{array}$ & $\begin{array}{c}.0942844 \\
(.3436534)\end{array}$ & $\begin{array}{c}.0575972 \\
(.3446657)\end{array}$ & $\begin{array}{c}-.1927812 \\
(.3538438)\end{array}$ & $\begin{array}{l}-.3015495 \\
(.3568697) \\
\end{array}$ & $\begin{array}{l}.0165441 \\
(.40689) \\
\end{array}$ & $\begin{array}{c}.0550188 \\
(.4074949)\end{array}$ \\
\hline$O D I$ & & $\begin{array}{c}.0047207 * * * \\
(.0016277)\end{array}$ & $\begin{array}{c}.0043892 * * * \\
(.0016414)\end{array}$ & $\begin{array}{c}.0025741 \\
(.0017412)\end{array}$ & $\begin{array}{c}.0016621 \\
(.0017804)\end{array}$ & $\begin{array}{c}.0013594 \\
(.0017941)\end{array}$ & $\begin{array}{l}.0011144 \\
(.001802)\end{array}$ \\
\hline \multicolumn{8}{|c|}{$P R O[$ AGRI/ FARM for reference] } \\
\hline EMPEE & $\begin{array}{r}-.3069187 \\
(.7253675) \\
\end{array}$ & $\begin{array}{l}-.3462591 \\
(.7248855) \\
\end{array}$ & $\begin{array}{l}-.2828602 \\
(.7315173) \\
\end{array}$ & $\begin{array}{l}-.3529243 \\
(.7205122) \\
\end{array}$ & $\begin{array}{l}-.2468833 \\
(.7290824) \\
\end{array}$ & $\begin{array}{l}-.1964959 \\
(.7346422) \\
\end{array}$ & $\begin{array}{l}-.2029376 \\
(.7345013) \\
\end{array}$ \\
\hline UNSKIL & $\begin{array}{c}-.7074268^{* * *} \\
(.2368527) \\
\end{array}$ & $\begin{array}{c}-.6896878^{* * *} \\
(.2379471) \\
\end{array}$ & $\begin{array}{c}-.6695259 * * * \\
(.2383764) \\
\end{array}$ & $\begin{array}{c}-.6520184^{* * *} \\
(.2389762) \\
\end{array}$ & $\begin{array}{c}-.6146274 * * * \\
(.2396764)\end{array}$ & $\begin{array}{c}-.6316204 * * * \\
(.2399496) \\
\end{array}$ & $\begin{array}{c}-.6332986 * * * \\
(.2399474) \\
\end{array}$ \\
\hline SEMISKIL & $\begin{array}{l}-.4588799 \\
(.4504533)\end{array}$ & $\begin{array}{l}-.4763712 \\
(.4497339) \\
\end{array}$ & $\begin{array}{l}-.440043 \\
(.449716) \\
\end{array}$ & $\begin{array}{c}-.481517 \\
(.4478465) \\
\end{array}$ & $\begin{array}{l}-.4252077 \\
(.4471466)\end{array}$ & $\begin{array}{l}-.3866172 \\
(.4452744) \\
\end{array}$ & $\begin{array}{l}-.3883025 \\
(.4456338) \\
\end{array}$ \\
\hline SKILLED & $\begin{array}{c}-.5167978 \\
(.536011) \\
\end{array}$ & $\begin{array}{l}-.5584367 \\
(.5402944) \\
\end{array}$ & $\begin{array}{l}-.524674 \\
(.540977) \\
\end{array}$ & $\begin{array}{l}-.5557367 \\
(.546665) \\
\end{array}$ & $\begin{array}{l}-.5008143 \\
(.5477293) \\
\end{array}$ & $\begin{array}{l}-.5136517 \\
(.5493584) \\
\end{array}$ & $\begin{array}{l}-.5182881 \\
(.5495787) \\
\end{array}$ \\
\hline PROF & $\begin{array}{l}-.1675311 \\
(.8277892) \\
\end{array}$ & $\begin{array}{r}-.1486558 \\
(.828731) \\
\end{array}$ & $\begin{array}{l}-.0131553 \\
(.8321238) \\
\end{array}$ & $\begin{array}{l}-.2735697 \\
(.8413358) \\
\end{array}$ & $\begin{array}{l}-.0669505 \\
(.8444997) \\
\end{array}$ & $\begin{array}{c}.0404454 \\
(.8484995) \\
\end{array}$ & $\begin{array}{l}-.0011086 \\
(.8528796) \\
\end{array}$ \\
\hline OTHER & $\begin{array}{l}-.7138298 \\
(.5783829) \\
\end{array}$ & $\begin{array}{c}-.6863493 \\
(.5792479) \\
\end{array}$ & $\begin{array}{l}-.6538148 \\
(.5797166) \\
\end{array}$ & $\begin{array}{l}-.5691152 \\
(.5797068) \\
\end{array}$ & $\begin{array}{l}-.5029617 \\
(.5811192) \\
\end{array}$ & $\begin{array}{l}-.4270688 \\
(.5811098) \\
\end{array}$ & $\begin{array}{c}-.39149 \\
(.5826008) \\
\end{array}$ \\
\hline$O D I$ & & $\begin{array}{c}.0045131^{* * *} \\
(.0016257) \\
\end{array}$ & $\begin{array}{c}.0042508^{* * *} \\
(.0016372) \\
\end{array}$ & $\begin{array}{l}.0028338^{* *} \\
(.0017118) \\
\end{array}$ & $\begin{array}{c}.0022139 \\
(.0017392)\end{array}$ & $\begin{array}{c}.0012252 \\
(.0017948)\end{array}$ & $\begin{array}{c}.0009661 \\
(.0018036)\end{array}$ \\
\hline Prov Dummy & YES & YES & YES & YES & YES & YES & YES \\
\hline Year Dummy & YES & YES & YES & YES & YES & YES & YES \\
\hline Obs & 1214 & 1214 & 1214 & 1214 & 1214 & 1214 & 1214 \\
\hline Wald chi2 & 18.34 & 21.49 & 26.28 & 35.91 & 40.59 & 33.49 & 42.26 \\
\hline Pseudo $\mathrm{R}^{2}$ & 0.38 & 0.48 & 0.62 & 0.65 & 0.69 & 0.55 & 0.61 \\
\hline
\end{tabular}

Note: Numbers in the "()" is robust standard error ; *** **, * indicate significant at $1 \%, 5 \%$ and $10 \%$ respectively.

\section{Conclusion}

China's ODI and the Chinese residents' happiness is positively correlated, ie, China's ODI is conducive to the improvement of domestic happiness. The income increase has little effect on the happiness of people in home country, and the Gini coefficient, the inflation rate and the unemployment rate decrease considerably the effect of ODI on the happiness of the people in home country, and the negative effect by Gini coefficient is higher than inflation and umployment.

The happiness coefficient of different groups were different. Among those of significant coefficients, the coefficient of the ODI's effect on happiness of the medium and high educated, the single, the divorced, housewives, the very-healthy and the relatively-healthy is positive; the happiness of the unemployed, the annual income between 20000-50000, the unskilled workers is negatively correlated with ODI.

The the trend of effect of ODI on the happiness of different populations is also different, but most of them is deteriorated. The happiness of the medium and high educated, the single, housewife, the relatively-healthy and the fair-healthy, the very healthy, the unskilled worker are slightly worsen because of the ODI. The happiness of the divorced, the groups of income of between 20000-50000 are improved by ODI. 


\section{Acknowledgements}

This study is supported by the following projects:

National Science Foundation of Guangdong Province, On the Effect of Inflation on the Efficiency of the Real Economy, 2014A030313624;

Characteristic Innovation Project of Guangdong Colleges and Universities, Investigation and Study on the Development Path of the Daguanghai Gulf marine industry;

Youth Foundation of Wuyi University, On the Employment Effect of Overseas Transfer of Industry based on the background of “One Belt and One Road” Strategy, 2015sk04.

\section{References}

[1] Li Lei, Bai Daohuan, Xian Guoming. How FDI Affect Employment? -- a Study Based on Chinese Micro Enterprise Data[J]. Economic Research, 2016(8):144-158.

[2] Ren Zhicheng. Study on the Employment Effect of International Industrial Transfer[M]. Economic Science Press,2012:55.

[3] Mao Qilin, Xu Jiayun. How does China's FDI Affect Employee Income? [J]. Industrial Economic Research,2014,(06):50-59.

[4] Gazaniol A. Does Outward Foreign Direct Investment affect domestic real wages? An investigation using French micro-data[J]. Fiw Working Paper, 2015.

[5] Yuan Qigang, Shang Hui, Zhang Wei. An Analysis of the Mechanism of FDI on Wage [J]. World Economy Research,2015,(11):80-89+128-129.

[6] Slaughter M J1 Multinational Corporations1 Outsourcing, and American Wage Divergence1 National Bureau of Economic Research (NBER) Working Paper, 5253

[7] Lee C G. Outward Foreign Direct Investment and Economic Growth: Evidence from Japan[J]. Global Economic Review, 2010, 39(3):317-326.

[8] Qi Jianmei, Wang Mingyi. FDI Expand the Enterprise Wage Gap between You -- Empirical Evidence Based on Micro Data in China[J]. International Trade Issue,2017,(01):116-126.

[9] Dunning J H. Explaining the International Direct Investment Position of Countries: Towards a Dynamic or Developmental Approach[J]. Review of World Economics, 1981, 117(1):30-64.

[10] Pichl C. Economic Development and Foreign Investment Linkages[J]. Wifo Monatsberichte, 1989, 62.

[11] Vining K. A Method to Identify Stages II and III in Dunning's Investment Development Path[J]. Dissertations \& Theses - Gradworks, 2016.

[12] Divarci A, Hisarciklilar M, Kayalica M O, et al. Foreign Direct Investment and Development in MENA Countries[J]. Middle East \& North Africa, 2005.

[13] Yang Jianquan, Yang Xiaowu, Wang Jie. An Empirical Study on FDI in China: IDP Test and Trend Analysis[J]. International Trade Issues , 2006(8):76-81.

[14] Gorynia M, Nowak J, Wolniak R. Foreign direct investment of Central and Eastern European countries, and the investment development path revisited[J]. Eastern Journal of European Studies, 2010, 1(2):21-36.

[15] Virmani S, Amann E. Is the evolution of Indiaâ€ $€^{\mathrm{TM}}$ S Outward FDI consistent with Dunningâ€ ${ }^{\mathrm{TM}_{S}}$ Investment Development Path sequence?[J]. Working Papers, 2015.

[16] Zhao Yan, Huang Weiwei. An Empirical Study on the Relationship Between Foreign Direct Investment and Inflation [J]. Enterprise Economy, 2013 (3): 157 - 160.

[17] Zhou Jinlong, Chu Shasha. An Empirical Study on the Relationship Between FDI and Macroeconomic Variables in China[J]. Time Finance, 2015(6X):13-14. 
[18] Kanweri. Understanding the Impact of Foreign Direct Investment on the Happiness of Countries[D]. https://thesis.eur.nl/pub/33849

[19] Tella R D, Macculloch R. Happiness, Contentment and Other Emotions for Central Banks[J]. Social Science Electronic Publishing, 2007.

[20] Jiang Qiping. From the Digital Divide to the Happiness Gap -- a Review of "Huang Youguang" Happiness Gap in East Asia[J]. Internet Weekly, 2003(43):72-73. 